Des êtres vivants et des artefacts

\title{
Inauguration du colloque
}

Frédéric Keck

\section{(2) OpenEdition}

\section{Journals}

Édition électronique

URL : http://journals.openedition.org/actesbranly/652

ISSN : 2105-2735

Éditeur

Musée du quai Branly Jacques Chirac

Référence électronique

Frédéric Keck, "Inauguration du colloque », Les actes de colloques du musée du quai Branly Jacques Chirac [En ligne], 6 | 2016, mis en ligne le 20 janvier 2016, consulté le 08 septembre 2020. URL : http:// journals.openedition.org/actesbranly/652

Ce document a été généré automatiquement le 8 septembre 2020

(c) Tous droits réservés 


\title{
Inauguration du colloque
}

\author{
Frédéric Keck
}

Bonjour à tous.

2 Je suis très heureux d'inaugurer ce colloque portant sur les êtres vivants et les artefacts, c'est-à-dire sur l'imbrication des processus vitaux et des processus techniques.

3 Nous avons trois types de colloques au musée du quai Branly : des colloques portant sur les grandes figures de l'histoire de l'anthropologie et de l'histoire de l'art ; des colloques sur les grandes aires culturelles, en général à l'occasion des expositions qui ont lieu ici ; et des colloques sur des thématiques, comme celle-ci, sur lesquelles nous souhaitons encourager des recherches, notamment celles qui sont menées par les boursiers à l'intérieur de cette institution. Le colloque qui va se tenir ici pendant deux jours fait partie de ces orientations thématiques qui ne portent pas forcément sur un sujet de société, mais sur un sujet qui est au cœur des enjeux des disciplines qui sont illustrées ici : anthropologie, archéologie, histoire de l'art.

4 Pour illustrer ces trois colloques et faire un exercice un peu artificiel de transition, comme dans un séminaire où on essaie de montrer qu'il y a une unité entre les différentes activités et les différentes personnes qu'on reçoit, je voudrai faire le lien entre ce colloque et celui qui vient d'avoir lieu autour d'Emmanuel Terray, et les suivants, qui vont porter sur les plumes et les Indiens des Plaines. Donc, des sujets qui apparemment n'ont rien à voir.

$5 \quad$ Le colloque qui vient d'avoir lieu sur Emmanuel Terray essaie de revenir aux grands débats de l'anthropologie marxiste des années 1960, qui ont été un grand moment de créativité dans la pensée française. On a repris les notions de mode de production, de reproduction, et de domination. Il me semble que le couple conceptuel que nous proposent Ludovic Coupaye et Perig Pitrou - que je remercie pour leur proposition stimulante - permet d'interroger à nouveau cette question de la production et de la domination. Si on regarde l'opposition entre êtres vivants et artefacts, c'est l'une des grandes oppositions ontologiques que l'on essaie de déconstruire aujourd'hui en anthropologie, comme celle qui passe depuis Lévi-Strauss entre nature et culture ; mais si on regarde plus précisément, et si je comprends bien l'intention qui est celle de 
Ludovic et de Perig, l'idée est de prendre êtres vivants et artefacts comme des étapes ou des résultats d'un processus de production, de croissance et de décroissance, d'émergence et de dégénérescence, dont ils sont des aspects. On aurait tendance à penser que l'artefact est ce sur quoi les sociétés humaines ont une prise, puisqu'elles en sont l'origine, alors que la vie serait extérieure à l'activité humaine. Je relisais pour l'occasion Art and Agency d'Alfred Gell, où il dit que seul le géologue peut imaginer ce que serait que fabriquer une montagne. De la même façon, quand on est face aux êtres vivants, on est réceptif à leur émergence, à leur croissance spontanée, alors que pour l'artefact, on pense en être à l'origine. En fait, un ensemble de phénomènes qui vont être au cœur de ce colloque brouille cette opposition entre la vie qui serait une émergence spontanée et l'artefact qui serait de création humaine.

6 Pour vous donner un exemple - ce serait la transition vers le prochain colloque, la question des plumes, et généralement la question des objets dans un musée -, les objets qui figurent dans un musée sont clairement des artefacts, même s'ils n'ont pas toujours des noms d'auteur. Mais ils peuvent également être considérés comme des êtres vivants au sens où, d'abord, il y a des parties organiques dans les objets d'un musée : on voit, par exemple dans l'exposition «Bois sacrés ", les composants organiques d'un masque. Les artefacts peuvent aussi être considérés comme des êtres vivants au sens où ils trouvent, par exemple dans l'espace d'un musée, quelque chose comme une seconde vie, c'est-à-dire qu'il faut penser les conditions dans lesquelles ils sont conservés, restaurés, au-delà des conditions dans lesquelles ils étaient initialement prévus pour durer.

7 Le colloque qui va avoir lieu sur les plumes posera la question de savoir jusqu'à quel point on peut restaurer des plumes qui font partie d'une parure. À quel moment une plume fait partie d'un artefact qu'il faudrait conserver comme un objet d'art ? À quel moment est-ce que sa dimension organique, vivante, donc capable de dégénérescence resurgit dans les actes par lesquels on les restaure ? Questions que l'on se pose également quand on voit l'affiche de l'exposition Indiens des Plaines. L'artefact frappe par sa capacité à manifester des aspects des êtres vivants.

8 A travers cette thématique qui va nous permettre de couvrir un vaste ensemble de phénomènes humains, on est aussi au cœur des problèmes qui se posent dans un musée lorsqu'on doit considérer des artefacts comme des êtres vivants.

Je vous remercie pour l'organisation de ce colloque et je me réjouis de le suivre.

\section{AUTEUR}

\section{FRÉDÉRIC KECK}

Frédéric Keck a étudié les dispositifs de surveillance des maladies animales transmissibles aux humains au Laboratoire d'anthropologie sociale et est directeur du département de la recherche et de l'enseignement au musée du quai Branly. Il est notamment l'auteur d'Un monde grippé (Flammarion, 2010) et il a co-dirigé (avec Noëlie Vialles) Des hommes malades des animaux (Éditions 
de L'Herne, 2012).

[Frederic.Keck [@] quaibranly.fr] 